\title{
Application of Simultaneous Localization of Multiple Pulmonary Nodules in a Hybrid Operating Room for Uniportal Video-Assisted Thoracic Surgery
}

\author{
Qi Zhang' \\ Zhiqiang Wang' \\ Ying Liu ${ }^{2}$ \\ Huajie Xing ${ }^{\prime}$ \\ Jian $X u^{\prime}$ \\ Wei Xu' \\ Huarong Cai' \\ Yuequan Jiang' \\ 'Chongqing Key Laboratory of \\ Translational Research for Cancer \\ Metastasis and Individualized Treatment, \\ Chongqing University Cancer Hospital, \\ Chongqing, People's Republic of China; \\ ${ }^{2}$ Youyang Tujia and Miao Autonomous \\ County Center for Disease Control and \\ Prevention, Chongqing, People's Republic \\ of China
}

\begin{abstract}
Objective: This study explores the value of the application of simultaneous localization of multiple pulmonary nodules in a hybrid operating room for uniportal video-assisted thoracic surgery (VATS).

Methods: This study performed a retrospective analysis of 60 patients with multiple pulmonary nodules (the number of nodules in every patient was $\geq 2,131$ in total) admitted to our hospital from September 2020 to September 2021. After computerized tomography (CT) scanning in a hybrid operating room, a multi-hook locating needle was used for simultaneous localization. The localization success, surgical resection, and locating needle unhooking rates of multiple pulmonary nodules were analyzed. The complication incidence, localization time, operation time, anesthesia time, post-isolation nodule search time, and postoperative hospital stay length were analyzed. In addition, the patients' anxieties about the puncture localization were evaluated.

Results: The intraoperative CT scans successfully showed all pulmonary nodules. The localization success, unhooking, and nodule resection rates were 98.5\% (129/131), 1.5\% $(2 / 131)$, and $100 \%(131 / 131)$, respectively. The median times of the localization, operation, anesthesia, post-isolation pulmonary nodule search, and hospital stay were 19 min [interquartile range (IQR): 15-30 $\mathrm{min}$ ], $98 \mathrm{~min}$ (IQR: 80-110 $\mathrm{min}$ ), $149.5 \mathrm{~min}$ (IQR: 126-171 $\mathrm{min}$ ), $3.5 \mathrm{~min}$ (IQR: $1-5 \mathrm{~min}$ ), and $6 \mathrm{~d}$ (IQR: $4-9 \mathrm{~d}$ ), respectively. The incidences of pneumothorax and pulmonary hemorrhage were $20.0 \%(12 / 60)$ and $13.3 \%(8 / 60)$, respectively. The self-rating anxiety scale score of the patients was $53.6 \pm 6.1$.

Conclusion: The hybrid operating room could be beneficial in accurately localizing multiple pulmonary nodules with reasonable safety and patient tolerance, and it is applicable to uniportal VATS.
\end{abstract}

Keywords: hybrid operating room, intraoperative CT, multiple pulmonary nodules, uniportal thoracoscopy

\section{Introduction}

Video-assisted thoracic surgery (VATS) is a primary treatment method for the resection of pulmonary nodules. However, it is difficult to detect most pulmonary nodules during an operation. ${ }^{1}$ It is challenging in an uniportal VATS, and the palpation failure rate is about $54 \%{ }^{2}$ Even if pulmonary nodules could be excised successfully, it takes a long time to find them in isolated lung tissue. ${ }^{3}$ Thus, the accurate localization of pulmonary nodules is crucial to guide surgeons to find them 
quickly and remove them completely. This study primarily investigates the preoperative localization of multiple pulmonary nodules. In practical clinical work, there are more cases of multiple pulmonary nodules, many of which are multiple primary malignant pulmonary nodules. The localization of multiple pulmonary nodules is relatively complicated. Preoperative multi-needle puncture localization methods will increase injury; this is difficult for patients to accept, so they fail to cooperate well. Meanwhile, complications increase, affecting localization success and excision rates. Thus, thoracic surgeons face great challenges. From September 2020 to September 2021, the simultaneous localization of multiple pulmonary nodules combined with uniportal VATS in a hybrid operating room was conducted. This study examines the results of 60 patients, and the efficacy is satisfactory.

\section{Materials and Methods}

\section{Clinical Data}

The patients who underwent simultaneous localization for multiple pulmonary nodules in a hybrid operating room from September 2020 to September 2021 were retrospectively included in this study.

The inclusion criteria were as follows: $\geq 2$ ipsilateral pulmonary nodules; pure ground-glass nodules $\geq 5 \mathrm{~mm}$; solid or mixed density nodules $\geq 8 \mathrm{~mm}$; pulmonary nodules difficult to detect during an operation; and patients whose cardiopulmonary and other organ functions could tolerate surgery. The exclusion criteria included patients with nodules located in the hilum, patients with the puncture route running through large vessels, if the bony structure of the chest wall was obviously obscured, and if the patient had general conditions that could not tolerate surgery.

\section{Methods}

\section{Surgical Indications}

High risk nodules were absolute surgical indications. The definition of high-risk nodules: 1. Ground glass nodules over $8 \mathrm{~mm}$, especially those with mixed density; 2 . Ground glass nodules gradually increased in the follow-up process, or realistic components, or solid components increased; 3 . Ground glass nodules persisted for more than 1 year, and there was no reduction in follow-up. 4. Nodules with malignant signs in CT imaging, such as vascular penetration sign, burr sign, pleural pull sign, vacuole sign, etc. 5 . History of malignant tumors. For pulmonary nodules discovered for the first time, we suggest patients follow up for 3-6 months or even 1 year according to the different conditions of the nodules. If the above conditions are met, surgical resection will be recommended.

\section{Localization of Multiple Pulmonary Nodules}

Under general anesthesia, intubation was performed in the two lumens, and the appropriate position was selected according to the location of the nodules. According to preoperative chest computerized tomography (CT) data, a fully covered metal localization grid was placed in the thorax, and a large-aperture sliding rail CT scan (Siemens, Germany; SOMATOM Confidence) was used in a hybrid operating room to confirm the skin puncture point of each nodule and measure the puncture depth and angle, as is shown in Figure 1.

\section{Puncture of Multiple Pulmonary Nodules}

A disposable pulmonary nodule locating (multi-hook) needle (model: SS510-10 Senscure, China) was used for puncture (Figure 2). The anchor needle was "four-claw" circular arc, the puncture needle and push tube were made of medical stainless steel, and the positioning line was made of medical suture material (non-absorbable). The anchor needle is made of nickel-titanium memory alloy wire. When the hook is released, it will firmly grasp the adjacent lung tissue, even if it is inserted into small blood vessels will not cause bleeding, low decoupling rate. Two surgeons operated simultaneously and synchronously punctured multiple locating needles to the side of each nodule according to the puncture path. After pushing out the needle core, the push rod exited the puncture trocar and pulled out the connecting wire at the end of the locating needle. After the completion of localization, CT reexamination was conducted to confirm the positional relationship between the locating needle and the nodule. In addition, pneumothorax, bleeding, unhooking, and other complications were examined, as is shown in Figure 3.

\section{Operation Method}

Under general anesthesia plus double-lumen endo-tracheal tube, the patient was placed in a 90-degree lateral position to the healthy side. After completion of disinfection and surgical drape laying, a 3-4 cm incision was made in the 4-5 intercostal space between the mid axilla and the front of the affected side, and an uniportal thoracoscopy was performed to detect the position of each locating mark and whether the locating needle was unhooked. According to the comprehensive judgment of the nodule size, shape, location, and lung function of the patients, pulmonary wedge resection, 


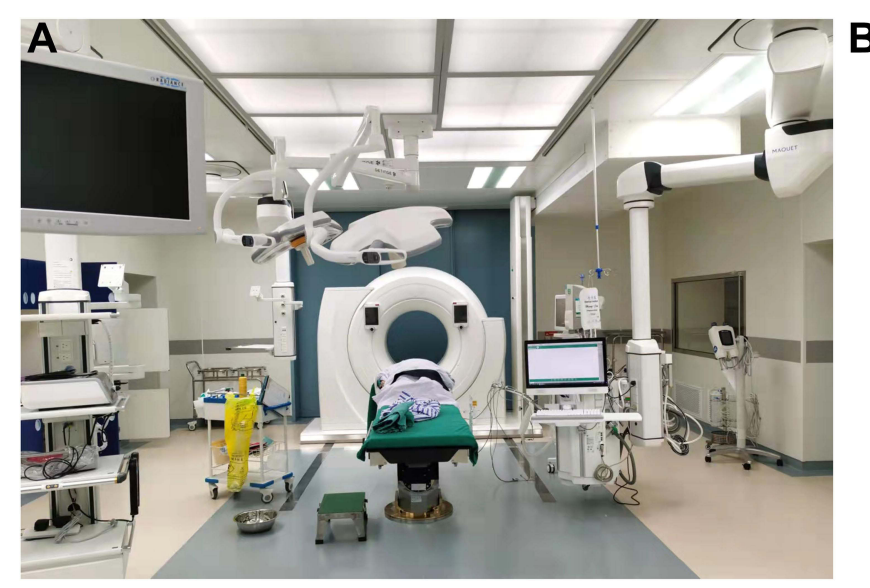

B

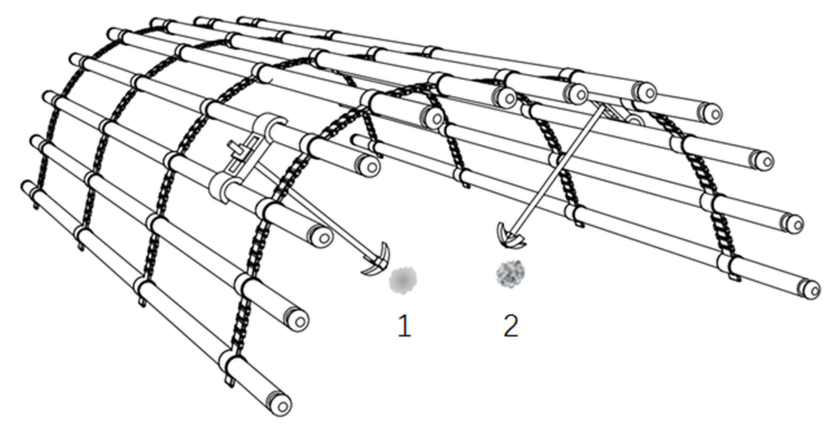

Figure I Localization of multiple pulmonary nodules in hybrid operating room. (A) Hybrid operating room and large-aperture sliding rail CT; (B) schematic diagram of simultaneous localization and puncture of pulmonary nodules I and 2 with full-coverage thoracic surface locating grid.

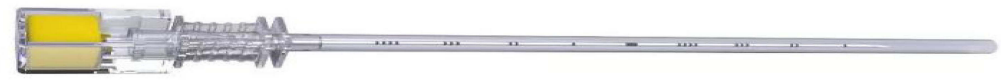

Multi-hook type Hook Wire (SS510-10 Senscure, China)

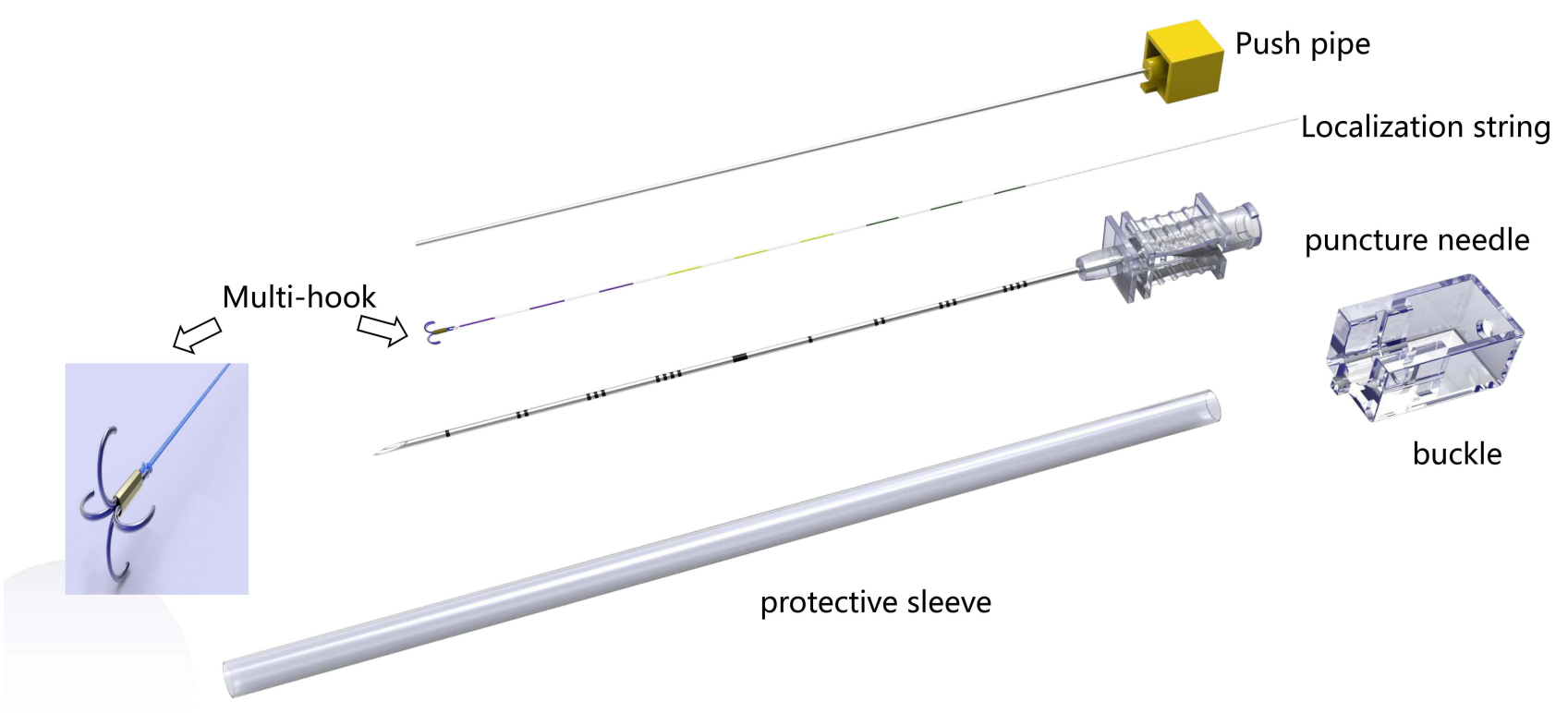

Figure 2 A disposable pulmonary nodule locating needle was used for puncture.

segmentectomy, or lobectomy under uniportal thoracoscopy was chosen. Lung tissues were taken out from the specimen bag, and pulmonary nodules were searched along the locating needle to judge the integrity of the locating needle and nodules. An intraoperative pathological examination was rapidly conducted to confirm the property of the nodule. In the case of invasive adenocarcinoma, a lobectomy combined with lymph node dissection was performed.

\section{Statistical Treatment}

The localization success, surgical resection, and locating needle unhooking rates of multiple pulmonary nodules were calculated. The incidence rates of complications (eg, pneumothorax and pulmonary hemorrhage) were calculated. Postoperative hospital stay length and localization, operation, anesthesia, and post-isolation nodule search times were recorded. The patients' anxiety about 

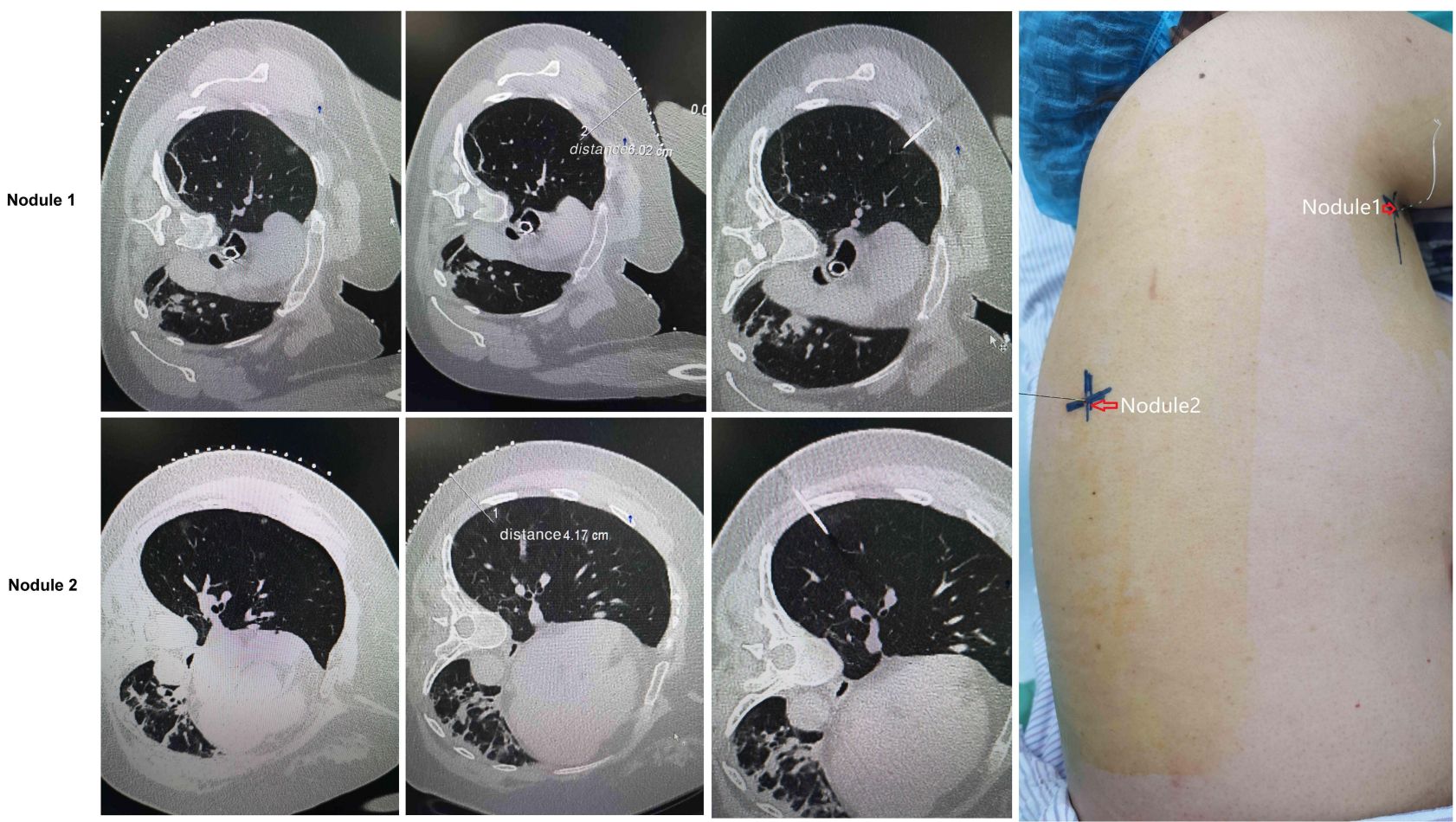

Nodule 2
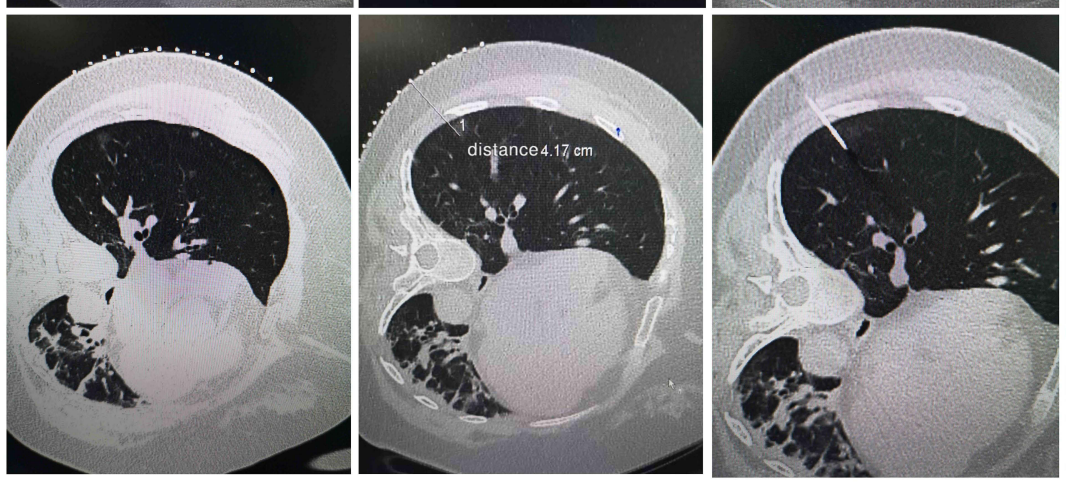

Figure 3 Simultaneous localization and puncture of right upper pulmonary nodule I and right lower pulmonary nodule 2 in hybrid operating room.

puncture localization was evaluated using the self-rating anxiety scale (SAS), where a score of 50-59 points indicated mild anxiety; 60-69 points indicated moderate anxiety; and more than 70 points indicated severe anxiety.

\section{Results}

In total, 60 patients were included in this study with 131 pulmonary nodules in total. Among them, 50 patients had two high-risk pulmonary nodules, and 10 patients had three high-risk pulmonary nodules. The average size of the nodules was $8.02 \pm 0.9 \mathrm{~mm}$, and the nodules were 3.47 $\pm 1.2 \mathrm{~mm}$ away from the pleura. The average patient age was $55 \pm 14.1$ years (Table 1).

All pulmonary nodules were examined by intraoperative frozen pathological. Two patients underwent lobectomy due to they had 2 nodules located in the same lobe and they were confirmed with primary invasive lung cancer by intraoperative frozen pathology. Besides, 56 cases underwent segmentectomy and 71 wedge resection. The postoperative pathology identified 88 were primary

Table I Observation Indicators and Results of Simultaneous Localization of Multiple Pulmonary Nodules in Hybrid Operating Room

\begin{tabular}{|l|l|l|l|}
\hline Average Age (y) & Number of Nodules (n) & Mean Size (mm) & $\begin{array}{l}\text { Mean Depth Away from the } \\
\text { Pleura (mm) }\end{array}$ \\
\hline $55 \pm 14.1$ & 131 & $8.02 \pm 0.9$ & $3.47 \pm 1.2$ \\
\hline Median localization time (min) & Median operation time (min) & Median anesthesia time (min) & Post-isolation nodule search time (min) \\
\hline 19 & 98 & 149.5 & 3.5 \\
\hline Median hospital stay time (d) & Localization success rate (\%) & Surgical resection rate $(\%)$ & Unhooking rate (\%) \\
\hline 6 & $98.5 \%$ & $100 \%$ & $1.5 \%$ \\
\hline Incidence of pneumothorax (\%) & Incidence of pulmonary bleeding (\%) & SAS score (points) & Proportion of malignancy (\%) \\
\hline $20.0 \%$ & $13.3 \%$ & $53.6 \pm 6.1$ & $72 \%$ \\
\hline
\end{tabular}


Table 2 The Operation Procedure and Final Pathological Results

\begin{tabular}{|c|c|}
\hline Parameters & $\mathbf{N}$ \\
\hline \multicolumn{2}{|l|}{ Operation procedure } \\
\hline Lobectomy & 4 \\
\hline Segmentectomy & 56 \\
\hline Wedge resection & 71 \\
\hline \multicolumn{2}{|l|}{ Final pathology } \\
\hline Primary lung cancer & 88 \\
\hline Lung metastases & 6 \\
\hline Benign nodule & 37 \\
\hline \multicolumn{2}{|l|}{ Type of primary malignant nodules } \\
\hline Atypical adenomatous hyperplasia & 17 \\
\hline Adenocarcinoma in situ & 21 \\
\hline Microinvasive adenocarcinoma & 42 \\
\hline Invasive adenocarcinoma & 8 \\
\hline
\end{tabular}

lung cancer nodules, 6 were lung metastases nodules, and 37 were benign nodules. Among the primary malignant nodules, 17 were atypical adenomatous hyperplasia, 21 were adenocarcinoma in situ, 42 were microinvasive adenocarcinoma, and 8 were invasive adenocarcinoma (Table 2). All pathological results showed negative resection margin. Systematic dissection was performed in 4 patients undergoing lobectomy, including $\mathrm{N} 1$ and N2. Selective lymph node sampling was only performed in the remaining patients, and no lymph node metastasis was found in all patients.

In this study, all pulmonary nodules were successfully displayed by intraoperative CT in a hybrid operating room. Among the 131 nodules, 129 were localized accurately (a localization success rate of $98.5 \%$ ). Two of the subpleural nodules showed locating needle loss (an unhooking rate of $1.5 \%$ ). Later, the 60 patients received an uniportal thoracoscopy, and all nodules were completely removed (a resection rate of $100 \%$ ). The median times for lesion localization, operation and anesthesia time, post-isolation pulmonary nodule search, and hospital stay were $19 \mathrm{~min}$ [interquartile range (IQR): 15-30 min], $98 \mathrm{~min}$ (IQR: 80$110 \mathrm{~min}$ ), $149.5 \mathrm{~min}$ (IQR: 126-171 $\mathrm{min}$ ), $3.5 \mathrm{~min}$ (IQR: 1-5 $\mathrm{min}$ ), and $6 \mathrm{~d}$ (IQR: 4-9 d), respectively. A small amount of pneumothorax occurred in 11 patients, and a moderate amount occurred in one patient [a pneumothorax incidence of $20.0 \%(12 / 60)]$. Eight cases showed a small amount of bleeding in the intrapulmonary needle passage [an intrapulmonary bleeding incidence of
$13.3 \%(8 / 60)]$. No severe bleeding occurred. The bleeding site was removed along with the pulmonary nodules. The patients' SAS scores were $53.6 \pm 6.1$, and the rate of postoperative malignant nodules was $72 \%$, as is shown in Table 1.

\section{Discussion}

With the application of high-resolution CT, enhanced awareness of physical examination, and the emergence of an aging population, the detection rate of pulmonary nodules increases gradually, ${ }^{4}$ and many patients manifest with multiple pulmonary nodules. Some pulmonary nodules have a high risk of malignancy, ${ }^{5}$ and in particular, the ground-glass opacity malignancy probability is as high as $63 \%{ }^{6}$ Thus, more attention has been paid to the surgical treatment of multiple pulmonary nodules. The accurate localization of multiple pulmonary nodules is key to the success of the surgery. If they fail to be localized, doctors will be forced to adopt palliative resections, extend resection protocols, or even perform a second operation, all of which will adversely affect patients.

Traditional intraoperative anatomical, tactile pressure sensing, and ultrasonic localizations have a high failure rate, ${ }^{7}$ so the classical preoperative CT puncture localization method is often used to locate pulmonary nodules. However, current research primarily focuses on single pulmonary nodules. ${ }^{8,9}$ This method is implemented step by step, as follows: after localization in the CT room, the hook-wire locating needle is inserted; the patient is then transferred to the operating room for surgery. The preoperative waiting time is uncertain. During the waiting period, the risk of pneumothorax and bleeding increases, both of which can easily cause locating needle loss. Moreover, CT room rescue conditions are limited. Once complications occur in puncture localization, emergency treatment is required, and doctors are often in a passive position. Clinical findings show that complications are more apparent when more pulmonary nodules are localized. To avoid this, Chao et $\mathrm{al}^{10}$ used intraoperative localization of pulmonary nodules in a hybrid operating room. It achieved good effects and reduced the risk of complications. However, multiple pulmonary nodules were not studied in this method. Some domestic scholars used preoperative CT for the simultaneous localization of multiple pulmonary nodules and achieved good results on the whole, but they still could not avoid the risk of puncture complications and the heavy psychological burdens to the patients. $^{11}$ Currently, there are few studies on single 
nodule localization in hybrid operating rooms in China. This study's results indicate that the hybrid operating room was used to successfully achieve the simultaneous localization of multiple pulmonary nodules and avoid a series of disadvantages of preoperative puncture localization technology. ${ }^{12,13}$ After anesthesia, this method was implemented by two surgeons simultaneously to ensure the effective puncture and accurate localization of each nodule and the resection of all pulmonary nodules at once. The results show that the technique had a high success rate of localization and nodule resection, with a low incidence of complications, equivalent to the preoperative CT localization of single nodules. Due to accurate localization, surgeons can quickly locate each nodule in the lung tissue and reduce surgery and anesthesia times. In this study, locating needle loss was found in two nodules in thorax exploration. This was not caused by the puncture technique but was due to the location of the two nodules (below the pleura) and the fact that the locating needle was too shallow. After the puncture, the connecting wire was fixed in vitro, and an intraoperative pneumothorax resulted in unhooking due to the pull of the connecting wire for localization; during the operation, remedial localization was performed to locate the unhooked pulmonary nodules through hemorrhagic pleural points, and they were completely resected. Thus, for patients with subpleural nodules combined with emphysema, it is recommended that the puncture needle be placed 1-2 $\mathrm{cm}$ deep in the nodules to reduce the probability of unhooking. In this study, there was little evidence of pneumothorax and intrapulmonary bleeding after a puncture, so they did not cause any difficulty in anesthesia and surgery.

Preoperative CT locating puncture is an invasive operation performed in the waking state of patients, and patients generally have a great fear of the procedure. The high tension and anxiety during the puncture and the pain and chest discomfort after results in patients having a very poor treatment experience. ${ }^{14}$ This study evaluated puncture localization in a hybrid operating room after general anesthesia, and the results showed that the SAS score was $53.6 \pm 6.1$, indicating mild anxiety. Patients reported no uncomfortable experience caused by the punctures. This method, combined with uniportal VATS, causes minor trauma, few complications, and a short hospital stay. In addition, the patients' satisfaction with the treatment is significantly improved.

In this study, the incidence of pneumothorax was $20 \%$. All pneumothorax occurred in our study were small amount of pneumothorax with lung compression no more than $10 \%$, which was limited to the local thorax. The possible reasons were as follow: 1. Patients with emphysema are more likely to develop pneumothorax; 2 . The deeper nodules with multiple nodules are prone to pneumothorax; 3. Intraoperative positive pressure ventilator ventilation may increase the inducement of pneumothorax; 4. In this study, all the patients included were multiple nodules. Compared with single nodules, puncture sites increased and the incidence of pneumothorax increased. However, it has been reported in literatures that the incidence of hookwire locating pneumothorax is $21.6 \%-38 \%{ }^{15-17}$ The incidence of pneumothorax in this study is lower than that reported in the literatures. Except for one case of decoupling caused by moderate amount of pneumothorax, the occurrence of a small amount of pneumothorax did not affect the positioning accuracy and patients.

\section{Conclusion}

In conclusion, simultaneous localization of multiple pulmonary nodules in a hybrid operating room combined with uniportal thoracoscopy technology perfectly combines precision and minimal invasion. This "one-step" operation mode can easily achieve the localization and resection of multiple pulmonary nodules. This method is highly safe and effective and is easily acceptable to patients.

\section{Ethics Approval and Consent to Participate}

I confirm that I have read the Editorial Policy pages. This study was conducted with approval from the Ethics Committee of Chongqing University Cancer Hospital (No: CZLS2021042-A). This study was conducted in accordance with the declaration of Helsinki. Written informed consent was obtained from all participants.

\section{Consent for Publication}

All participants signed a document of informed consent.

\section{Funding}

Chongqing Science and Health Joint Medical Research

Project 2021. General program (No: 2021MSXM214).

\section{Disclosure}

The authors declare that they have no competing interests. 


\section{References}

1. Seo JM, Lee HY, Kim HK, et al. Factors determining successful computed tomography-guided localization of lung nodules. $J$ Thorac Cardiovasc Surg. 2012;143(4):809-814. doi:10.1016/j.jtcvs.2011.10.038

2. Suzuki K, Nagai K, Yoshida J, et al. Video-assisted thoracoscopic surgery for small indeterminate pulmonary nodules: indications for preoperative marking. Chest. 1999;115(2):563-568. doi:10.1378/ chest.115.2.563

3. Mattioli S, D'Ovidio F, Daddi N, et al. Transthoracic endosonography for the intraoperative localization of lung nodules. Ann Thorac Surg. 2005;79(2):443-449. doi:10.1016/j.athoracsur.2004.07.087

4. Weinstock T, Kidambi P, Channick CL, et al. Implementation of Lung Cancer Screening Programs with Low-Dose Computed Tomography in Clinical Practice. Ann Am Thorac Soc. 2016;13(3):425-427. doi:10.1513/AnnalsATS.201512-804CME

5. Wahidi MM, Govert JA, Goudar RK, Gould MK, McCrory DC; American College of Chest Physicians. Evidence for the treatment of patients with pulmonary nodules: when is it lung cancer?: ACCP evidence-based clinical practice guidelines (2nd edition). Chest. 2007;132(3 Suppl):94S-107S. doi:10.1378/chest.07-1352

6. Migliore M, Fornito M, Palazzolo M, et al. Ground glass opacities management in the lung cancer screening era. Ann Transl Med. 2018;6 (5):90. doi:10.21037/atm.2017.07.28

7. Zhang L, Xie D, Chen C. [Application progress of intraoperative localization of pulmonary ground glass nodules]. Zhong Guo Xiong Xin Xue Guan Wai Ke Lin Chuang Za Zhi. 2018;25(12):1080-1084. Chinese.

8. Park $\mathrm{CH}$, Han $\mathrm{K}$, Hur J, et al. Comparative effectiveness and safety of preoperative lung localization for pulmonary nodules: a systematic review and meta-analysis. Chest. 2017;151(2):316-328. doi:10.1016/j. chest.2016.09.017

9. Wang WH, Yu T. [Research progress on common localization methods of pulmonary nodules in thoracoscopic surgery]. Mod Oncol. 2019;27 (22):4111-4115. Chinese.
10. Chao YK, Pan KT, Wen CT, Fang HY, Hsieh MJ. A comparison of efficacy and safety of preoperative versus intraoperative computed tomography-guided thoracoscopic lung resection. $J$ Thorac Cardiovasc Surg. 2018;156(5):1974-1983.e1. doi:10.1016/j.jtcvs.2018.06.088

11. Li MM, Li Z, Shen J, Mao GC, Zhang S, Ni CF. [The application of simultaneous Hook-wire multi-needle localization technique in thoracoscopic resection of multiple pulmonary nodules]. $J$ Intervent Radiol. 2020;29(11):1136-1139. Chinese.

12. Chao YK, Pan KT, Wen CT, Fang HY, Hsieh MJ. Preoperative CT versus intraoperative hybrid DynaCT imaging for localization of small pulmonary nodules: a randomized controlled trial. Trials. 2019;20(1):400. doi:10.1186/s13063-019-3532-z

13. Mazza F, Venturino M, Peano E, et al. Single-stage localization and thoracoscopic removal of nonpalpable pulmonary nodules in a hybrid operating room. Innovations (Phila). 2020;15(6):555-562. doi:10.1177/1556984520961039

14. Zhao ZR, Lau RWH, Yu PSY, Ng CSH. Devising the guidelines: the techniques of pulmonary nodule localization in uniportal video-assisted thoracic surgery-hybrid operating room in the future. $J$ Thorac Dis. 2019;11(Suppl 16):S2073-S2078. doi:10.21037/ jtd.2019.01.82

15. Li FW, Chen YT, Bian JW, et al. [Preliminary study on the localization of micro coil guided by CT before operation of multiple pulmonary nodules]. Chin J Lung Cancer. 2018;21:857-863. Chinese. doi:10.3779/j.issn.1009-3419.2018.11.08

16. Huang XY, Zheng YF, Pan F, et al. [Application value of double hook wire localization under CT guidance before thoracoscopic resection of small pulmonary nodules]. Jie Ru Fang She Xue Za ZHi. 2017;26:1098-1101. Chinese.

17. Zhong Y, Xu XQ, Chen L, et al. [The guiding value of COMBINED Hookwire and methyblue localization under $\mathrm{CT}$ guidance for thoracoscopic multiple pulmonary nodules resection.] Yi Xue YIng Xiang Xue Za Zhi. 2017;27:667-669. Chinese.
International Journal of General Medicine

\section{Publish your work in this journal}

The International Journal of General Medicine is an international, peer-reviewed open-access journal that focuses on general and internal medicine, pathogenesis, epidemiology, diagnosis, monitoring and treatment protocols. The journal is characterized by the rapid reporting of reviews, original research and clinical studies across all disease areas. The manuscript management system is completely online and includes a very quick and fair peer-review system, which is all easy to use. Visit http://www.dovepress.com/ testimonials.php to read real quotes from published authors. 